\section{Clinical gist}

\author{
John Launer
}

This is a tale of a medical muddle. Almost certainly, you will have heard similar tales from your own friends or relatives, or seen such things happening in your workplace. In this instance, I have combined several cases and altered details for anonymisation, but not exaggerated anything.

A 65 year-old woman went to the accident and emergency department of her local hospital one Friday morning, because she had swallowed a piece of fish the previous evening, and it got stuck. This had happened to her several times previously, because she suffers from an oesophageal stricture. The casualty officer who saw her, presumably hearing mainly the words "fish" and "stuck", and also more familiar with seeing patients who have accidentally swallowed fish bones, ordered a lateral $\mathrm{x}$-ray of the neck. This was normal. Because the woman was having chest discomfort, the doctor next asked for a wide range of urgent blood tests, and an ECG, which appeared to show possible atrial flutter. It had to be repeated a few times before a cardiologist was called to reassure everyone that this was an artefact, resulting from the fact that the woman also suffers from early Parkinson's disease and has a tremor.

Now that a retained fish bone and a cardiac event had been excluded, the accident and emergency doctor called the on-call ear, nose and throat doctor to come and assess the patient. Unfortunately, the ENT team were all in the operating theatre or busy on the wards, so it took a while for anyone to arrive. By now, the patient had not been able to eat and drink for nearly 24 hours, and had received very little in the way of intravenous fluids. Different nurses came and went, but since this was a casualty department, no individual nurse had been allocated to care for the patient or monitor her general condition, so she was now dehydrated. A young doctor from the ENT department did eventually come, saying he had been called in to look into the patient's throat.

Correspondence to Dr John Launer, Faculty Development, Health Education England, Stewart House, 32 Russell Square, London WC1B 5DN, UK: john.launer@nwl.hee.nhs.uk
At this point, the woman explained (as she had already tried to do with the other doctors) that the obstruction seemed to be further down than the throat, in exactly the same place that had occurred in previous incidents. She pointed her finger quite precisely at the lower end of her sternum. "Then you don't come under our department at all", the doctor pronounced. "I'll have to call the duty gastro-enterologist." Unfortunately it was very late in the day, and the gastro-enterology team had already gone home for the weekend. A hospital admission over the weekend under the acute medical team was by then inevitable. It took until the following Monday afternoon for her to have an oesophageal dilatation.

\section{FANTASTICALLY SIMPLE}

Stories of patient care like this, full of false turns and dead ends, are so familiar that it sometimes appears as if they have become the norm. They arise from the convergence of many different kinds of problem, at several levels of the system. At the individual level, the casualty officer in this case probably took an inadequate history, failed to consult the past notes, and did not consider bringing in a senior doctor, who might have known more about this patient's chronic condition. At the level of clinical reasoning, the doctors all displayed some well-established cognitive errors, including the tendency to assume a common rather than an unual condition ("availability error") and not re-examining their original judgement in the light of emerging findings ("anchoring error"). ${ }^{1}$ In terms of the overall organisation of the hospital, there was a lack of continuity in the medical and nursing care, with no clear ownership of the patient, fragmentation between five different specialties, and inflexible shift working. $^{2}$ Looking at modern medical culture generally, one can spot the familiar tendencies of doctors to practise defensively, to try and "rule everything out", and to carry out multiple investigations rather than hold a conversation with patients, relatives or each other. ${ }^{3}$ Yet when all is said and done, the case was a fantastically simple one: here is a woman with a tight gullet who has swallowed a lump of food too big to go through to her stomach.
An intelligent school child with no knowledge of medicine might have realised this. The vast resources of a large general hospital, taken together, were more of an impediment than an aid to the simple process of getting the "clinical gist."

The concept of clinical gist is given little prominence in medical education and practice, although it almost certainly should be. Farrell Lloyd from the Mayo Clinic, and his behavioural scientist colleague Valerie Reyna have addressed it directly. Writing in the Journal of the American Medical Association, they emphasise its importance in medical decision-making. ${ }^{4}$ They draw a helpful distinction between "verbatim representations" of information, and "gist representations". The former are laid down in memory as literal facts, whereas the latter capture the meaning or interpretation of those facts. Inculcating gist memories in learners and practitioners, they argue, requires quite a different process of instruction from rote recall - one that emphasises "far transfer." This is essentially the ability to solve problems through simple pattern recognition, rather than the laborious consideration of multiple facts. Helping learners to extract the gist of cases systematically, they point out, has the advantage that gist memories endure over time and are more robust to interference from distractions. Applied to the case above, for example, a simple rubric like "if food gets stuck, it's probably a blockage in the oesophagus" would have trumped all the doctors' combined knowledge of radiology, electrocardiology, blood markers and everything else that got in the way of their thinking clearly.

Focussing on clinical gist would do more than prevent delay and inconvenience to patients. As a study in Sheffield in the United Kingdom has shown, there is a direct relationship between the efficiency of patient flow through systems like emergency rooms and the resulting mortality rate, not to mention financial costs. ${ }^{5}$ A lack of "gist thinking" may be lifethreatening both in a direct way, through delays in diagnoses and treatment, and in an indirect way, because it entails such massive wastage of human and medical resources. This suggests a compelling case for raising the profile of clinical gist in medical teaching and practice very significantly - though explicit training, good role modelling and, probably most of all, with the judicious use of patient narratives to demonstrate the nature and frequency of the problem. In the words of one 
specialist registrar writing recently in the BMJ: "Doctors should be trained to become confident in stating singularly what they think is going on. To achieve this... requires mostly only two things to take a history, and to think." 6

Twitter Follow John Launer at @JohnLauner

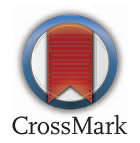

To cite Launer J. Postgrad Med J 2016;92:121-122.

Postgrad Med J 2016:92:121-122.

doi:10.1136/postgradmedj-2015-133938

\section{REFERENCES}

1 Groopman J. How Doctors Think. Boston: Houghton Miffllin, 2007.

2 Guthrie B, Saultz J, Freeman GK, Haggerty J. Continuity of care matters: relationships between doctors and patients are central to good care. BMJ 2008;337:a867.

3 Heath I. Role of fear in overdiagnosis and overtreatment. BMJ 2014;349:96123.

4 Lloyd F J, Reyna VF. Clinical gist and medical education: Connecting the dots. JAMA 2009; 302:1332-3.

5 The Health Foundation. The story of Flow, Cost, Quality in Sheffield. 14 Sept 2012. http://www.health. org.uk/story-flow-cost-quality-sheffield (accessed 22 Dec 2015)

6 Freudenthal B. We must reclaim the "Art of medicine". Rapid response. BMJ, 9 Nov 2014. http:// www.bmj.com/content/349/bmj.g6123/rr/779615 (accessed 22 Dec 2015) 